\title{
Kinetic Study of Thrombogenesis in Stroke-prone Spontaneously Hypertensive Rats (SHRSP)
}

\section{(2) Platelet Economy from 6 Weeks to 12 Months of Age under the Development of Hypertension}

\author{
Shigeki Koganemaru, Junko Nakagawa-Suzuki, \\ Hajime Okita, and Atsushi Kuramoto
}

We have reported that the platelet production increase in stroke-prone SHR (SHRSP) by means of increased incorporation of ${ }^{75} \mathrm{Se}$-selenomethionine into megakaryocytes and circulating platelets, is probably due to increased platelet consumption as compared with those in stroke resistant SHR (SHRSR) (Koganemaru S et al : Jap Heart J 18:598, 1977, Thrombos Hemostas (Stuttgart) $38: 139$, 1977).

In the present study, we extended our study on the advanced aged groups, to see if this kinetic change was brought about by vascular alteration per se or hemodynamic changes caused by developing hypertension.

Materials and Methods:

$\operatorname{SHRSP}\left(\mathrm{A}_{1}-\mathrm{sbF}_{37-39}\right)$ and $\operatorname{SHRSR}\left(\mathrm{B}_{1} \mathrm{~F}_{36-38}\right)$ with matched ages (5 weeks, 10 weeks, and 9-12 months) were used. At 10 weeks of age, SHRSP and SHRSR were divided in 4 groups to compare the maximum uptake of ${ }^{75} \mathrm{Se}$-selenomethionine. The first group was separated as a control. The second group was given $1 \% \mathrm{NaCl}$ solution for 5 weeks from 6 to 10 weeks of age. The third group was treated by anti-hypertensive drug : clonidine (Catapres) $15 \mathrm{\mu g} / \mathrm{Kg}$, once/day, i.p. for 4 weeks, from 7 to 10 weeks of age. The last group was treated by an anti-platelet agent : dipyridamole, $10 \mathrm{mg} / \mathrm{Kg}$, twice/day, i.p. for 1 week from 9 to 10 weeks of age. ${ }^{75} \mathrm{Se}-$ selenomethionine, with specific activity $54 \mathrm{mCi} / \mathrm{mg}$ was obtained from Commissariat a l'energie atomique, France. A dose of $2 \mu \mathrm{Ci} / 100 \mathrm{Gm}$ was injected via the tail vein. The rats were sacrificed and blood samples were obtained daily after radioisotope infusion up to 6 th day, from abdominal aorta with $1.5 \%$ EDTA. The platelets were separated by differential centrifugation and were washed once with saline. Gamma emission radioactivity from samples were counted by an Automatic Gamma Scintillation System Model 4224 (Nuclear Chicago).

Results:

Platelet kinetic study of SHRSP and SHRSR was followed from 6 weeks to 12 months of age. Maximum uptake of radioactivity by platelets was observed at 3rd-4th day in both groups at 6 weeks of age. At 9-10 weeks, the peak was on 3rd day in both groups and in SHRSP at 9-12 months of age. The platelet survival time was 4.9 days in both groups at 6 weeks of age, but at $9-10$ weeks, it was shorter in SHRSP, 3.7 days, than in SHRSR, 4.8 days. This tendency was more marked

From the Department of Medicine, Research Institute for Nuclcar Medicine and Biology, Hiroshima University, Hiroshima 734. 


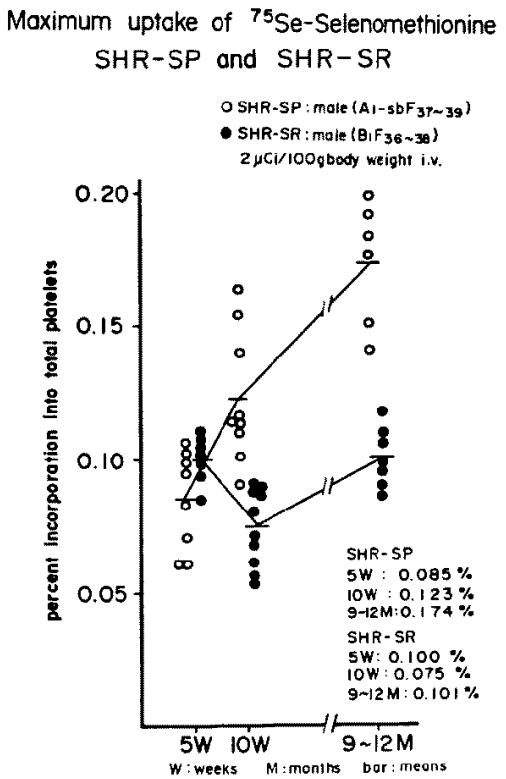

in SHRSP at 9-12 months. Maximum values of percent incorporation into total platelets were not so different between SHRSP and SHRSR at 5 weeks $(0.085 \pm$ 0.014 vs $0.100 \pm 0.010$, mean $\pm S D, N S)$. But it was higher in SHRSP compared with in SHRSR at 10 weeks of age $(0.123 \pm 0.022$ vs $0.075 \pm 0.011, \mathrm{p}<0.01)$. Furthermore at 9-12 months of age, it was remarkably increased in SHRSP, but not so changed in SHRSR $(0.174 \pm 0.023$ vs $0.101 \pm 0.014, \mathrm{p}<0.01)$ (Figure.).

The comparison of maximum uptake of ${ }^{75} \mathrm{Se}$-selenomethionine in various treatment groups versus the control group was done at 10 weeks of age. In the salt loading group, hypertension was accelerated from 188 to $205 \mathrm{mmHg}$ of the mean value but the maximum uptake was not affected $(0.123 \pm 0.022$ vs $0.124 \pm 0.030, \mathrm{NS})$. In the clonidine (Catapres) treated group, blood pressure decreased from 188 to 140 $\mathrm{mmHg}$ of the mean value, but maximum uptake did not decrease in SHRSP $(0.123$ \pm 0.022 vs $0.114 \pm 0.024$, NS). A significant decrease of the maximum uptake was observed in the dipyridamole treated group $(0.096 \pm 0.020, \mathrm{p}<0.05)$. Platelet adhesion to glass beads in this group decreased from $30 \%$ to $7.8 \%$ of the mean value, as well. On the other hand, there was no significant difference of maximum uptake among those groups in SHRSR.

\section{Conclusion :} SHRSP.

1) After 10 weeks of age, platelet production increased as the age advanced in

2) Salt loading to accelerate the development of hypertension, did not promote platelet consumption before 10 weeks of age.

3) Hypotensive drug :clonidine, did not suppress platelet production in spite of hypotensive effect in SHRSP.

4) Anti-platelet agent :dipyridamole suppressed platelet consumption in sustained hypertension.

This study was supported by the Science and Technology Agency of Japanese Government. 